\title{
La sierodiagnosi della toxoplasmosi nella gravida e nel neonato: esperienze dell'unità operativa dell'Azienda Sanitaria di Bolzano (Laboratorio di Microbiologia e Virologia)
}

\author{
Alessandra Marabese, Giacinto Anesi, Manuela Cia, Anna Gamper, \\ Elfrun Anna Maria Kafmann
}

Laboratorio di Microbiologia e Virologia, Azienda Sanitaria di Bolzano

Key Words: Toxoplasma gondii infection, serodiagnosis, pregnancy, congenital infection, immunoassays

The serodiagnosis of Toxoplasma gondii infection in pregnancies and newborns: experiences of Bolzano Hospital

\section{SUMMARY}

Serodiagnosis of $T$. gondii infection is difficult because the disease can manifest in several possible ways and because of the particolar dynamic of anticorpal response. The resulting interpretation problems entail prognostic, therapeutic and ethical difficulties in the case of pregnant women and their children. The resolution of these problems goes through an extremly articulate path: the information provided by screening tests intersects with results of supplementary assays and can be enriched thanks to complementary markers (IgA) and second-level tests (IgGavidity). Each laboratory must be able to choose and to combine complementary investigation methods and to plan an adequately diagnostic strategy. Sera arriving to our laboratory undergo screening by enzyme immunoassays Axsym Toxo lgG/lgM (Abbott). Subsequently confirmation of positive, weak positive and doubtful results is entrusted to the assays VIDAS Toxo IgGII/IgM (bioMérieux), and in case of newborns to the assay ETI-TOXO-MReverse Plus (diaSorin). IgA antibodies are determined by immunoassay ETI-TOXO-A-Reverse Plus (diaSorin); the IgG-avidity is evaluated by test Toxo-lgG-avidity (bioMérieux). Analysis of toxoplasma infections occured during pregnancyas resulting by a 3 years survey period, shows that combined use of our assays contribuites to a correct diagnosis. The contribution obtained by the comparison of evaluation IgGAxsym/IgG VIDAS, provides very useful clues to evaluate the infection degree in advances with respect to evaluation of IgG-avidity.

\section{RIASSUNTO}

La sierodiagnosi della toxoplasmosi incontra delle difficoltà legate alle diverse possibilità espressive della malattia, nonché alla peculiare dinamica della risposta anticorpale. Nelle gestanti e nei loro neonati, i problemi interpretativi che ne derivano implicano difficoltà di ordine prognostico, terapeutico ed etico. La loro risoluzione si snoda attraverso un percorso estremamente articolato: le informazioni dei test di screening si intersecano con i risultati di metodi di indagine supplementare e si arricchiscono grazie a marcatori complementari $(\operatorname{lgA})$ e ricerche di secondo livello (lgG-avidity). Ciascun laboratorio deve saper scegliere e combinare adeguatamente metodi di indagine complementari mettendo a punto percorsi diagnostici adeguati. I sieri che pervengono al nostro centro di afferenza vengono sottoposti ad uno screening utilizzando i test immunoenzimatici Axsym Toxo lgG/lgM (Abbott). I campioni risultati positivi, debolmente positivi o dubbi vengono ritestati tramite i dosaggi VIDAS Toxo IgGII/IgM (bioMérieux) e, nel caso delle IgM neonatali mediante il test ETI-TOXO-M-Reverse Plus (diaSorin). Le IgA vengono dosate tramite il saggio ETI-TOXO-A-Reverse Plus (diaSorin); la definizione dell'avidità utilizza il test Toxo-lgGavidity (bioMérieux). L'analisi dei casi di toxoplasmosi gestazionale individuati nel corso di 3 anni di osservazione dimostra come l'uso combinato dei nostri saggi contribuisca in modo determinante alla formulazione della diagnosi. Particolarmente utile si è rivelato il contributo fornito dal confronto lgGAxsym/lgG VIDAS, il quale, anticipatamente rispetto alla determinazione dell'avidità, consente di inquadrare il grado di evoluzione dell'infezione.

\section{INTRODUZIONE}

La Toxoplasmosi è un'antropozoonosi ampiamente diffusa in natura: il suo agente eziologico, il Toxoplasma gondii, è un protozoo parassita intracellulare obbligato di numerosi vertebrati omeotermi, uomo compreso(3). Ad eccezione dei soggetti immunodepressi, la forma più grave della malattia toxoplasmica è quella congenita, che può verificarsi solo nel caso di una primoinfezione in gravidanza e consegue al passaggio per via transplacentare del protozoo dal sangue materno a quello fetale (15). L'incidenza dell'infezione da Toxoplasma gondii in gravidanza è stimata attorno a 1-6/1000 gestazioni (6). Il rischio di trasmissione verticale del parassita risulta tanto più elevato quanto più tardivo è il periodo gestazionale 
in cui viene contratta l'infezione (4). Le conseguenze dell'infezione risultano tanto più gravi quanto più precoce è il contagio materno: il protozoo può provocare aborto nelle prime settimane, lesioni e anomalie funzionali a carico degli occhi e del sistema nervoso centrale nel $2^{\circ}$ trimestre, nonché un'infezione sub clinica (con possibili sequele tardive oculari, uditive, nervose) nel $3^{\circ}(2,10,11)$. All'inizio della gravidanza le gestanti devono essere sottoposte (come previsto in Italia dal Decreto Ministeriale del 20/10/1998) ad uno screening sierologico (oggi per lo più affidato a test immunoenzimatici) finalizzato a valutare il grado di protezione immunologica nei confronti della malattia (9); in caso di negatività dello stesso le gestanti dovrebbero essere monitorate fino al termine della gravidanza allo scopo di individuare un'eventuale sieroconversione. I risultati ottenuti da un primo screening $\operatorname{IgG} / \operatorname{IgM}$ possono essere insufficienti nel definire il grado immunologico della paziente (12); ciò è in gran parte riconducibile al peculiare comportamento delle IgM specifiche, le quali, accanto ad un'evoluzione classica, in alcuni casi presentano una dinamica a breve (durata di 1 mese) o lunga (persistenza per 1 anno, talvolta anche 3-4 anni) permanenza, complicando così la definizione della fase esordiente della malattia $(13,15)$. Ulteriori difficoltà possono derivare dalla presenza di $\operatorname{IgM}$ aspecifiche, ma soprattutto di IgM naturali (5) particolarmente frequenti in gravidanza. Per risolvere i problemi interpretativi che ne derivano è utile associare al test di screening metodi di indagine supplementare da utilizzare a conferma di esiti dubbi o debolmente positivi, nonché avvalersi dell'utilizzo di marcatori sierologici complementari (IgA) (15) e di ricerche sierologiche di $2^{\circ}$ livello (IgG-avidity) $(7,14)$. Questo lavoro si prefigge lo scopo di illustrare le scelte metodologiche relative alla diagnosi di toxoplasmosi maturate presso il Servizio di Microbiologia e Virologia dell'Azienda Sanitaria di Bolzano; attraverso la presentazione di alcuni casi clinici saranno illustrate le difficoltà diagnostiche e prognostiche poste dalla toxoplasmosi e si dimostrerà come l'uso combinato dei nostri saggi, contribuisca alla formulazione della diagnosi.

\section{MATERIALI E METODI \\ Campioni}

Viene illustrata l'esperienza relativa all'analisi di 18740 sieri di donne gravide e 29 sieri di neonati da madri affette pervenuti al nostro laboratorio nel triennio 2002-2005 (Gennaio 2002-Gennaio 2005).

Test di screening: IgG e IgM Axsym

Tutti i sieri sono stati preliminarmente sottoposti ad uno screening $\operatorname{IgG} / \operatorname{IgM}$ utilizzando $\mathrm{i}$ test immunoenzimatici della ditta Abbott (Axsym Toxo IgG/Axsym Toxo IgM), dei saggi diretti, a cattura di microparticelle (tecnologia MEIA) eseguiti in maniera automatizzata sull'analizzatore Axsym. Il dosaggio Axsym Toxo IgG fornisce una determinazione anticorpale quantitativa, espressa in UI $/ \mathrm{ml}$. Titoli $>=3$ sono da considerare positivi, $<2$ negativi.

Il dosaggio Axsym Toxo IgM fornisce una determinazione anticorpale qualitativa. Il risultato viene espresso sotto forma di un indice. Un indice $>0.600$ è da considerare positivo, tra $0.500 \mathrm{e}$ 0.599 dubbio, $<0.499$ negativo.

Test di conferma: VIDAS Toxo IgGII - VIDAS Toxo IgM - ETI-TOXO-M-REVERSE-Plus DiaSorin,

I campioni risultati positivi, debolmente positivi o dubbi alla presenza di una o di entrambe le classi anticorpali sono stati sottoposti ad indagini di conferma; in particolare:

- La conferma dei risultati forniti dal dosaggio Axsym Toxo IgG è stata affidata al saggio Toxo IgGII della bioMérieux, un metodo qualitativo che associa la tecnica immunoenzimatica sandwich in 2 tappe ad una rivelazione finale in fluorescenza (ELFA). Analogamente ad Axsym, i risultati vengono espressi in $\mathrm{UI} / \mathrm{ml}$. Titoli $>=8$ sono da considerare positivi, tra 4 e 8 dubbi, $<4$ negativi.

- Per la conferma dei risultati forniti dal dosaggio Axsym Toxo IgM, disponiamo invece di 2 possibili saggi, la scelta dei quali varia in dipendenza della tipologia di paziente considerato:

a) nel caso di donne gravide (o soggetti sintomatici) con sospetta toxoplasmosi abbiamo utilizzato il dosaggio Toxo IgM della bioMérieux un metodo quantitativo che associa la tecnologia a cattura di tipo reverse ad una rivelazione finale in fluorescenza (ELFA). Analogamente ad Axsym i risultati vengono espressi sotto forma di un indice; un indice $>=$ 0.65 è da considerarsi positivo, tra 0.55 e 0.65 dubbio, $<0.55$ negativo.

b) nel caso di neonati da madri affette da toxoplasmosi abbiamo preferito affidare la conferma dei risultati Axsym IgM, siano essi positivi o negativi (la performance del dosaggio Axsym IgM non è stata stabilita per campioni di sangue neonatale) ed il monitoraggio di questa classe anticorpale al dosaggio ETI-TOXO-MREVERSE-Plus della DiaSorin, un saggio qualitativo a cattura di tipo reverse su micropiastra. A differenza dei dosaggi IgM sopraccitati, il saggio della DiaSorin rende visibile ad ogni seduta il valore del controllo cut-off ed 
esprime il risultato come rapporto tra l'assorbanza media del campione e l'assorbanza del controllo cut-off (un indice $>=1$ e da considerare positivo, $<1$ negativo). Ora considerando che il cut-off per la positività neonatale è generalmente più basso del 20\%-30\% rispetto all'adulto, la visibilità del controllo cut-off assicura indubbiamente una migliore valutazione della sierologia neonatale.

\section{Metodi di indagine supplementare: ETI- TOXO-A-Reverse-Plus DiaSorin - VIDAS Toxo IgG-avidity.}

Per la determinazione delle IgA abbiamo utilizzato il dosaggio ETI-TOXO-A-Reverse-Plus della

DiaSorin, un test immunoenzimatico a cattura di tipo reverse da eseguire su micropiastra. Il saggio fornisce una valutazione anticorpale semi-quantitativa, espressa in unità arbitrarie $(\mathrm{AU} / \mathrm{ml})$, come risultato dell'interpolazione del valore di assorbanza di ciascun campione su di una curva di taratura definita, ad ogni sessione, da una serie di 5 calibratori. Un titolo $>5$ è da considerarsi positivo, $<=5$ negativo.

La definizione dell'avidità è stata affidata al saggio Toxo IgG-avidity della bioMérieux, un saggio automatizzato sul sistema VIDAS. I risultati vengono espressi in termini di indice. Un indice < $0.200(20 \%)$ indica la presenza di $\operatorname{IgG}$ a debole avidità e suggerisce un'infezione acuta contratta non più di 3 mesi prima; un indice compreso tra $0.20(20 \%)$ e $0.30(30 \%)$ indica la presenza di IgG ad avidità intermedia e suggerisce un'infezione recente contratta nei 4-6 mesi precedenti; un indice $\geq=0.30(30 \%)$ indica la presenza di $\operatorname{IgG}$ a forte avidità e suggerisce un'infezione pregressa contratta nei 6 mesi precedenti.

\section{RISULTATI E DISCUSSIONE Considerazioni generali}

Dei 18740 sieri di donne gravide a noi pervenuti: l'11.5\% (2164) è risultata positiva alle sole IgG e quindi dotata di protezione immunitaria nei confronti della malattia, 1'88.3\% (16547 pazienti) è risultata negativa tanto alle IgG quanto alle IgM e quindi priva di protezione immunitaria, lo $0.15 \%$ (29 pazienti) è risultata positiva ad entrambe le classi anticorpali rivelando (come è stato poi accertato dai metodi di indagine supplementare) un'infezione toxoplasmica più o meno recente. Da una prima e sommaria analisi dei patterns immunitari relativi ai casi di toxoplasmosi individuati nella nostra popolazione di gestanti emergono alcune interessanti considerazioni (tabella 1).

- Anche nella nostra esperienza i risultati forniti dallo screening IgG/IgM non permettono di definire con sufficiente accuratezza il grado immunologico delle pazienti poiché ben il $65 \%$ di esse esprime le IgM ad oltre 6 mesi di distanza dall'esordio (figura IB), con picchi di permanenza che in alcuni casi raggiungono i 1642 mesi.

- Indicazioni aggiuntive relative allo stato immunologico delle gestanti sono emerse dalla determinazione delle IgA, le quali, nella maggior parte dei casi, a differenza delle IgM non sono risultate rilevabili ad oltre 6 mesi di distanza dall' esordio dell'infezione (figura IC).

- Tuttavia il maggior contributo alla determinazione del grado immunologico e della fase evolutiva dell'infezione è scaturita dalla determinazione dell'IgG-avidity (figura ID) e ancor prima, dal confronto tra il valore di IgG fornito dal dosaggio di screening della Abbott (Axsym Toxo IgG) ed il valore di IgG fornito dal dosaggio di conferma della bioMérieux (VIDAS Toxo IgGII). Abbiamo infatti verificato l'esistenza di una stretta correlazione tra l'avidità delle IgG e il DIgG Axsym/IgG VIDAS: valori IgG VIDAS notevolmente inferiori ai valori IgG Axsym si associano a percentuali di avidità inferiori al $20 \%$ e dunque ad infezioni acute; valori IgG VIDAS confrontabili o leggermente superiori ai valori IgG Axsym correlano a percentuali di avidità comprese tra il $20 \%$ e il $30 \%$ ovvero ad infezioni recenti; valori IgG VIDAS notevolmente superiori ai valori IgG Axsym si associano a percentuali di avidità maggiori del $30 \%$ quindi ad infezioni pregresse. Rispetto al dosaggio Axsym, il saggio della bioMérieux ci risulta dunque meno sensibile alla presenza delle IgG neoformate. Considerando che i due dosaggi sfruttano lo stesso principio (test immunoenzimatico diretto) utilizzando sia diluenti, sia tamponi di lavaggio, che un tracciante enzimatico identici (anticorpi monoclonali anti IgG umane marcati con fosfatasi alcalina) la differente sensibilità sembra essere ragionevolmente riconducibile alla diversa natura dell'antigene utilizzato: per il saggio VIDAS, la bioMérieux dichiara di impiegare antigene toxoplasmico di membrana e citoplasmatico (ceppo RH di Sabin), mentre per il dosaggio Axsym, la Abbott afferma di utilizzare microparticelle rivestite di antigene di $T$. gondii. La minor sensibilità alla presenza di IgG neoformate conferisce al dosaggio VIDAS un valore diagnostico che va ben oltre il ruolo del test di conferma: seppur sommariamente il dosaggio IgG VIDAS, associato al test Axsym, fornisce delle informazioni relative al grado di evoluzione dell'infezione, anticipatamente rispetto alla determinazione dell'IgG-avidity permettendoci peraltro di smascherare casi di 
toxoplasmosi relativi a quadri sierologici di per sé non sospetti.

\section{Casi clinici interessanti:donne in gravidanza}

L'analisi e la gestione diagnostica dei casi di toxoplasmosi emersi nel corso di questi ultimi 3 anni ha posto alla nostra attenzione patterns immunitari e quadri sierologici particolari, indubbiamente esemplificativi delle possibilità espressive della malattia. Particolare interesse ha suscitato il caso di una gestante nella quale abbiamo riscontrato un'infezione toxoplasmica caratterizzata da una mancata (o rapida) evoluzione della risposta IgM. Il caso, illustrato in tabella 2, dimostra come l'uso combinato e ragionato di test diagnostici complementari ha rivelato un'infezione, presumibilmente contratta all'inizio della gestazione, a fronte di un quadro che classicamente avrebbe autorizzato a considerare la gestante immune alla toxoplasmosi. Forte di questa evidenza il medico curante ha potuto inserire la gestante in un programma terapeutico adeguato a base di rovamicina preservandola così dalle possibili gravi conseguenze fetali.

Come accennato in precedenza abbiamo anche rilevato diverse situazioni caratterizzate dalla permanenza delle IgM oltre la fase acuta. A titolo esemplificativo riportiamo uno di questi casi (tabella 3) dal quale emerge che l'utilizzo dei nostri saggi ha permesso di identificare la fase avanzata di un'infezione contratta in epoca periconcezionale, anche se il quadro clinico indicava una toxoplasmosi acuta. Dato il rischio pressoché nullo di trasmissione verticale, la gestante non è stata sottoposta ad alcuna terapia medica, ma è stata monitorata con visite ecografiche che non hanno rivelato malformazioni o disturbi dello sviluppo fetale.

Dalla descrizione di questi due casi emerge anche come la determinazione delle IgA non abbia fornito delle informazioni fondamentali per la definizione del grado immunologico delle nostre pazienti, a dispetto dell'impatto risolutivo esercitato in primis dal DIgG Axsym/IgG VIDAS, e successivamente dalla determinazione dell'IgGavidity. Peraltro, l'individuazione di casi di mancata produzione di IgA e di situazioni di prolungata permanenza di tali anticorpi, unitamente al fatto che le IgA rimangono dosabili indiscriminatamente nella fase acuta così come in quella recente dell'infezione, ha notevolmente ridimensionato il nostro interesse nei confronti di questo marcatore. Esemplificativo è in questo senso il caso clinico riportato in tabella 4: esso descrive un'infezione toxoplasmica contratta nei 6 mesi precedenti, caratterizzata da IgM residue e da un'ingannevole lunga permanenza delle IgA che si protrae oltre le IgM anche nei mesi successivi.
L'elevata percentuale di avidità delle IgG consente di collocare la sieroconversione in epoca periconcezionale e autorizzando così a non sottoporre la propria paziente ad alcuna terapia. Alla luce di queste evidenze abbiamo deciso di limitare la determinazione delle IgA ai casi in cui il basso valore delle $\operatorname{IgG}($ VIDAS Toxo $\operatorname{IgG} \mathrm{II}<5$ ) non consente di definire l'avidity, nonché ai quadri sierologici $\mathrm{IgG}^{-} / \mathrm{IgM}^{+}$ove, in attesa di un secondo prelievo, si pone il problema di discriminare tra la presenza di anticorpi naturali e la fase esordiente di una toxoplasmosi acquisita (le IgA conservano invece un importante valore diagnostico nella sorveglianza post-natale della toxoplasmosi congenita).

\section{Casi clinici interessanti: neonati da madri affette}

Per quanto riguarda i neonati da madri affette da toxoplasmosi, dall'analisi dei patterns sierologici e dalla consultazione delle cartelle cliniche, emerge un quadro piuttosto rassicurante: nessuno dei bambini ha mostrato alla nascita sintomi o segni clinici riferibili all'infezione (risultato comunque atteso, visto che nel $90 \%$ dei casi anche un neonato infetto risulta asintomatico), e inoltre, la maggioranza di essi non ha rivelato forme subcliniche o latenti della malattia. Costituisce eccezione il caso di un neonato nato da madre con toxoplasmosi accertata attorno alla $26^{\circ}$ settimana di gravidanza e adeguatamente trattata con rovamicina fino al termine della gestazione. Alla nascita il bambino appare sano e reattivo e come atteso il toxo test rivela la sola presenza di IgG, con IgM e IgA negative. Tuttavia, in attesa del responso definitivo derivante dal monitoraggio delle $\mathrm{IgG}$, la datazione della sieroconversione materna in un periodo successivo alla $24^{\circ}$ settimana di gestazione suggerisce di associare al follow-up un protocollo terapeutico (a base di Pirimetamina e Sulfadiazina, con aggiunta di acido folinico), finalizzato a prevenire le possibili complicanze tardive di un'eventuale infezione subclinica. I risultati del monitoraggio sierologico sono riportati in tabella 5 ed illustrano come la disponibilità di un valido dosaggio supplementare delle $\operatorname{IgM}$ (diaSorin) abbia precocemente svelato (Axsym IgM si è mantenuto negativo) come, a dispetto dell'adeguata terapia materna, il protozoo sia riuscito ad attraversare la barriera placentare instaurando nel feto un'infezione subclinica. Dalla consultazione della cartella clinica è emerso che, a circa 15 giorni di distanza dall'inizio del trattamento, l'insorgenza di una primitiva depressione midollare indotta dai farmaci aveva imposto la somministrazione di Pirimetamina e Sulfadiazina per brevi periodi di tempo a cui face- 
vano seguito sospensioni di circa 30 giorni (necessarie per garantire la risalita dei neutrofili). Probabilmente la necessaria periodica interruzione della terapia neonatale, unitamente alla concomitante e progressiva diluizione plasmatica della Rovamicina precedentemente somministrata alla madre, hanno indirettamente favorito la proliferazione del parassita, al quale il bambino ha prontamente reagito iniziando a montare un'attiva risposta immunitaria.

\section{CONCLUSIONI}

Questo breve rapporto sulla nostra esperienza illustra come le scelte metodologiche e i percorsi diagnostici adottati presso il nostro centro di afferenza, hanno permesso di inquadrare correttamente lo stato immunologico dei nostri pazienti (soprattutto gravide e neonati), di individuare quadri sierologici classicamente non sospetti $\left(\mathrm{IgG}^{+} / \mathrm{IgM}^{-}\right)$e di chiarire situazioni immunitarie fuorvianti guidando il medico verso una corretta gestione clinica.

In particolare:

- Lo screening eseguito sul sistema Axsym dimostra una sufficiente sensibilità nel fornire un preliminare inquadramento sierologico del paziente e consente di selezionare rapidamente i campioni che debbono essere sottoposti ad ulteriori indagini.

- L'utilizzo del sistema VIDAS permette di ritestare i campioni risultati positivi, debolmente positivi o dubbi alla presenza di una o di entrambe le classi anticorpali e di ottenere informazioni aggiuntive. Infatti, il saggio
VIDAS Toxo IgM risulta più specifico e meno sensibile rispetto al dosaggio Axsym alla presenza di IgM aspecifiche, consentendo così di individuare eventuali false positività dovute a fattori interferenti (frequenti soprattutto nelle gravide); il saggio VIDAS Toxo IgG II si rivela invece meno sensibile rispetto al dosaggio Axsym alla presenza di IgG neoformate, cosicché il confronto tra i risultati forniti dai 2 metodi (D Axsym/VIDAS) fornisce al laboratorista delle informazioni preliminari relative al grado di evoluzione dell'infezione.

- L'inquadramento temporale della sieroconversione rimane comunque affidato alla determinazione dell'IgG-avidity, parametro sierologico che vanta la maggior capacità di indicare la fase della malattia.

- Nelle gravide, e più in generale in tutti i soggetti tranne i neonati da madri affette, la determinazione delle $\operatorname{IgA}$ non sembra aggiungere informazioni fondamentali per la definizione del grado immunologico del paziente, fornendo, in alcuni casi, indicazioni addirittura fuorvianti; la loro utilità si limita alla risoluzione di quadri sierologici $\operatorname{IgG}^{-} / \operatorname{IgM}^{+}$e a casi in cui l'IgG-avidity non sia determinabile.

- Infine, nei neonati da madri affette da toxoplasmosi, è particolarmente utile affidare il monitoraggio delle $\operatorname{IgM}$ ad un saggio immunoenzimatico che includa, in ogni seduta, oltre al controllo positivo e negativo, un controllo cut-off. La visibilità del valore cut-off consente di giungere ad una più corretta valutazione della sierologia neonatale.

Tabella I. Patterns immunitari relativi ai casi di toxoplasmosi in gravidanza individuati nella nostra popolazione (N.D.= non determinabile)

\begin{tabular}{|c|c|c|c|c|c|c|}
\hline $\mathbf{N}^{\circ}$ CASO & $\begin{array}{c}\text { IGG AVIDITY } \\
<20 \% \text { INF.ACUTA } \\
20-30 \% \text { INF. RECENTE } \\
>30 \% \text { INF. PREGRESSA }\end{array}$ & $\begin{array}{c}\text { IGM AXSYM } \\
<0.499 \text { NEG } \\
0.500-0.599 \text { DUBBIO } \\
>0.600 \text { POS } \\
\end{array}$ & $\begin{array}{l}\text { IGM VIDAS } \\
1<0.55 \text { NEG } \\
0.55-0.65 \text { DUBBIO } \\
\mid>0.65 \text { POS }\end{array}$ & $\begin{array}{l}\text { IGG AXSYM } \\
<2 \text { U/ML NEG } \\
>3 \text { U/ML POS }\end{array}$ & $\begin{array}{l}\text { IGG VIDAS } \\
<4 \text { NEG } \\
4-8 \text { DUBBIO } \\
>8 \text { POS }\end{array}$ & $\begin{aligned} & \text { IGA SORIN } \\
> & 5 \text { AU/ML POS } \\
< & 5 \text { AU/ML NEG }\end{aligned}$ \\
\hline 1 & $10 \%$ & 0.799 & 0.75 & 44.4 & 10 & 7 \\
\hline 2 & N.D. & 0.83 & 0.74 & 187 & 5 & 3 \\
\hline 3 & N.D. & 3.89 & 4.96 & 11.9 & 1 & 90 \\
\hline 4 & N.D. & 4.22 & 3.25 & 671 & 7 & 115 \\
\hline 5 & $3 \%$ & 1.28 & 1.72 & 96 & 45 & 97 \\
\hline 6 & $4 \%$ & 0.621 & 0.59 & 157 & 24 & 16 \\
\hline 7 & $2 \%$ & 4.6 & 5.7 & 92 & 52 & 10 \\
\hline 8 & $9 \%$ & 4.8 & 5.9 & 709 & 332 & 156 \\
\hline 9 & $8 \%$ & 1.049 & 0.814 & 267 & 223 & 58 \\
\hline 10 & $10 \%$ & 0.645 & 0.721 & 125 & 86 & 23 \\
\hline 11 & $13 \%$ & 0.802 & 0.76 & 280 & 219 & 5 \\
\hline 12 & $13 \%$ & 0.411 & 0.32 & 32 & 33 & 15 \\
\hline 13 & $14 \%$ & 1 & 1.27 & 51 & 53 & 48 \\
\hline 14 & $1.6 \%$ & 1.25 & 1.47 & 287 & 72 & 25 \\
\hline 15 & $6 \%$ & 0.505 & 0.34 & 130 & 22 & 22 \\
\hline 16 & $17 \%$ & 1.136 & 1.29 & 275 & 249 & 35 \\
\hline 17 & $26 \%$ & 0.784 & 0.92 & 35 & 47 & 10 \\
\hline 18 & $29 \%$ & 1.138 & 1.15 & 539 & 786 & 29 \\
\hline 19 & $25 \%$ & 1.224 & 1.92 & 143 & 86 & 38 \\
\hline 20 & $21 \%$ & 0.788 & 0.71 & 73 & 193 & 40 \\
\hline 21 & $29 \%$ & 0.829 & 1.07 & 1100 & 1168 & 37 \\
\hline 22 & $47 \%$ & 0.807 & 1.18 & 38 & 76 & 3 \\
\hline 23 & $67 \%$ & 0.595 & 0.98 & 29 & 91 & 0 \\
\hline 24 & $50 \%$ & 0.629 & 0.850 & 74 & 144 & 3 \\
\hline 25 & $31 \%$ & 1.17 & 1.17 & 219 & 394 & 2 \\
\hline 26 & $33 \%$ & 0.605 & 1.21 & 190 & 220 & 6 \\
\hline 27 & $35 \%$ & 0.685 & 0.60 & 94 & 124 & 12 \\
\hline 28 & $58 \%$ & 0.683 & 0.700 & 40 & 100 & 2 \\
\hline 29 & $46 \%$ & 0.88 & 0.900 & 30 & 51 & 2 \\
\hline
\end{tabular}


Tabella 2. Pattern immunitario relativo al $l^{\circ}$ caso di toxoplasmosi descritto.

Alla $15^{\circ}$ settimana di gestazione, il primo Toxo-test della paziente rivela la positività delle lgG con lgM negative. L'assenza di lgM, peraltro confermata dal dosaggio Toxo lgM eseguito sul VIDAS suggerisce l'esistenza di una protezione immunologia nei confronti della zoonosi, ma il test di conferma delle $\lg G$ ci insospettisce fornendo un valore proporzionalmente inferiore rispetto ad Axsym e suggerendo che le lgG della paziente siano in gran parte di recente formazione. Ad oltre I mese di distanza (nel frattempo la paziente non intraprende alcuna terapia), le IgM risultano ancora negative mentre le lgG mostrano un discreto incremento; tale movimento anticorpale rafforza ulteriormente lipotesi di un'infezione toxoplasmica acuta la quale trova conferma definitiva nella positività delle lgA nonché nella ridotta percentuale di avidità.

\begin{tabular}{|c|c|c|c|c|c|c|}
\hline DATA & $\begin{array}{r}\text { IGG AXSYM } \\
<2 \text { U/ML NEG } \\
>3 \text { U/ML POS }\end{array}$ & $\begin{array}{l}\text { IGG } \\
\text { VIDAS } \\
<4 \text { NEG } \\
\text { 4-8 DUBBIO } \\
>8 \text { POS }\end{array}$ & $\begin{array}{c}\text { IGM AXSYM } \\
<0.499 \text { NEG } \\
0.500-0.599 \\
\text { DUBBIO } \\
>0.600 \text { POS }\end{array}$ & $\begin{array}{l}\text { IGM VIDAS } \\
1<0.55 \text { NEG } \\
0.55-0.65 \text { DUBBIO } \\
1>0.65 \text { POS }\end{array}$ & $\begin{array}{c}\text { IGA SORIN } \\
>5 \text { AU/ML } \\
\text { POS } \\
<5 \text { AU/ML } \\
\text { NEG }\end{array}$ & $\begin{array}{c}\text { IGG AVIDITY } \\
<20 \% \text { INF.ACUTA } \\
20-30 \% \text { INF. } \\
\text { RECENTE } \\
>20 \% \text { INF. } \\
\text { PREGRESSA }\end{array}$ \\
\hline $19 / 08 / 2002$ & 32 & 12 & 0.411 & 0.32 & & \\
\hline $23 / 09 / 2002$ & 64 & 33 & 0.428 & 0.38 & 15 & $14 \%$ \\
\hline $16 / 11 / 2002$ & 64 & 35 & 0.360 & 0.27 & & $19.7 \%$ \\
\hline
\end{tabular}

Tabella 3. Pattern immunitario relativo al $2^{\circ}$ caso di toxoplasmosi descritto .

Alla $12^{\circ}$ settimana di gravidanza,il primo Toxo-test della paziente rivela la positività di entrambe le classi anticorpali. I risultati dello screening trovano conferma nei dosaggi lgG/lgM eseguiti sul Vidas; tuttavia, il valore lgG fornito dal VIDAS si rivela proporzionalmente superiore rispetto al valore di Axsym suggerendo lipotesi di un'infezione toxoplasmica avanzata.Ad un $2^{\circ}$ prelievo di conferma eseguito a circa 2 settimane di distanza la situazione sierologia rimane pressoché immutata rafforzando ulteriormente lipotesi iniziale; la conferma definitiva viene tuttavia dalla determinazione delle lgA, le quali risultano assenti e, ancor più dalla definizione dell'lgG-avidity, stimata attorno al $31 \%$.

\begin{tabular}{|c|c|c|c|c|c|c|}
\hline DATA & $\begin{array}{l}\text { IGG AXSYM } \\
<2 \text { U/ML } \\
\text { NEG } \\
>3 \text { U/ML } \\
\text { POS }\end{array}$ & $\begin{array}{l}\text { IGG VIDAS } \\
\text { < } 4 \text { NEG } \\
\text { 4-8 DUBBIO } \\
>8 \text { POS }\end{array}$ & $\begin{array}{c}\text { IGM AXSYM } \\
<0.499 \text { NEG } \\
0.500-0.599 \\
\text { DUBBIO } \\
>0.600 \text { POS }\end{array}$ & $\begin{array}{c}\text { IGM VIDAS } \\
\text { I }<0.55 \text { NEG } \\
0.55-0.65 \\
\text { DUBBIO } \\
\text { I }>0.65 \text { POS }\end{array}$ & $\begin{array}{l}\text { IGA SORIN } \\
>5 \text { AU/ML } \\
\text { POS } \\
<5 \text { AU/ML } \\
\text { NEG }\end{array}$ & $\begin{array}{c}\text { IGG AVIDITY } \\
<20 \% \text { INF.ACUTA } \\
20-30 \% \text { INF. } \\
\text { RECENTE } \\
>20 \% \text { INF. } \\
\text { PREGRESSA }\end{array}$ \\
\hline $11 / 02 / 2003$ & 219.2 & 394 & 1.173 & 1.173 & & \\
\hline $24 / 02 / 2003$ & 187.9 & & 1.049 & & 3 & $31 \%$ \\
\hline
\end{tabular}

Tabella 4. Pattern immunitario relativo al $3^{\circ}$ caso di toxoplasmosi descritto.

Alla $10^{\circ}$ settimana di gestazione la paziente esegue il suo primo Toxo-test il quale evidenzia una discreta positività delle lgG in presenza di IgM debolmente positive. Il dosaggio VIDAS IgM non aggiunge indicazioni utili, fornendo anch'esso esito dubbio; decidiamo dunque di procedere alla determinazione delle IgA: il saggio della dia Sorin fornisce esito positivo e tale risultato sembra escludere il caso di una toxoplasmosi pregressa, propendendo invece per un'infezione più o meno recente. Questa diagnosi contrasta tuttavia con il dosaggio VIDAS di conferma delle lgG, il quale fornisce un valore superiore e non paragonabile o inferiore al risultato Axsym. Ad un $2^{\circ}$ prelievo di conferma eseguito a distanza di 14 giorni, la sierologia della paziente rimane pressoché immutata rendendo verosimile lipotesi di una toxoplasmosi pregressa. La conferma definitiva viene dalla determinazione dell'lgG-avidity, la quale risulta pari al 35\%

\begin{tabular}{|c|c|c|c|c|c|c|}
\hline DATA & $\begin{array}{c}\text { IGG } \\
\text { AXSYM } \\
<2 \text { U/ML } \\
\text { NEG } \\
>3 \text { U/ML } \\
\text { POS }\end{array}$ & $\begin{array}{c}\text { IGG VIDAS } \\
<4 \text { NEG } \\
4-8 \\
\text { DUBBIO } \\
>8 \text { POS }\end{array}$ & $\begin{array}{l}\text { IGM AXSYM } \\
<0.499 \text { NEG } \\
0.500-0.599 \\
\text { DUBBIO } \\
>0.600 \text { POS }\end{array}$ & $\begin{array}{c}\text { IGM VIDAS } \\
\text { I }<0.55 \text { NEG } \\
0.55-0.65 \\
\text { DUBBIO } \\
\text { I }>0.65 \text { POS }\end{array}$ & $\begin{array}{l}\text { IGA SORIN } \\
>5 \text { AU/ML } \\
\text { POS } \\
<5 \text { AU/ML } \\
\text { NEG }\end{array}$ & $\begin{array}{c}\text { IGG AVIDITY } \\
<20 \% \text { INF.ACUTA } \\
20-30 \% \text { INF. } \\
\text { RECENTE } \\
>20 \% \text { INF. } \\
\text { PREGRESSA }\end{array}$ \\
\hline $04 / 08 / 2003$ & 94.7 & 124 & 0.685 & 0.60 & 10 & \\
\hline $18 / 08 / 2003$ & 98.8 & & 0.624 & & 18 & $35 \%$ \\
\hline $03 / 11 / 2003$ & 74.7 & 108 & 0.564 & 0.61 & 7 & $31.3 \%$ \\
\hline
\end{tabular}

Tabella 5. Pattern immunitario relativo al $4^{\circ}$ caso di toxoplasmosi descritto (neonato).

Allo scadere del $2^{\circ}$ mese di vita le lgG anti-toxoplasma mostrano un sensibile calo fisiologico; non altrettanto rassicurante appare tuttavia il monitoraggio delle lgM: a dispetto del valore negativo emerso dal dosaggio di screening, il saggio della dia Sorin fornisce un valore di poco superiore al $30 \%$ del cut-off della seduta, ed un indice comunque maggiore rispetto a quello rilevato alla nascita. Le stesse IgA risultano mosse. Per quanto esiguo tale movimento anticorpale pone il sospetto di una possibile toxoplasmosi congenita. L'ipotesi trova conferma nei successivi controlli sierologici,: a 3 mesi di distanza dal precedente monitoraggio sierologico le lgM, dosate con il metodo della dia Sorin appaiono in ulteriore crescita. Nei mesi successivi la risposta lgM declina cedendo il posto all'ascesa delle IgG neonatali

\begin{tabular}{|c|c|c|c|c|}
\hline DATA & $\begin{array}{l}\text { IGG AXSYM } \\
<2 \text { U/ML NEG } \\
>3 \text { U/ML POS }\end{array}$ & $\begin{array}{c}\text { IGM AXSYM } \\
<0.499 \text { NEG } \\
0.500-0.599 \text { DUBBIO } \\
>0.600 \text { POS }\end{array}$ & $\begin{array}{l}\text { IGM SORIN } \\
\text { > I POS } \\
<\text { I NEG }\end{array}$ & $\begin{aligned} & \text { IGA SORIN } \\
> & 5 \text { AU/ML POS } \\
< & 5 \text { AU/ML NEG }\end{aligned}$ \\
\hline $18 / 09 / 2003$ & 507 & 0.050 & $0.045 / 0.453=0.099$ & 0 \\
\hline $16 / 10 / 2003$ & 186.7 & 0.093 & $0.090 / 0.250=0.360$ & 0 \\
\hline $26 / 11 / 2003$ & 45 & 0.085 & $0.262 / 0.340=0.829$ & 3 \\
\hline $06 / 02 / 2004$ & 150 & 0.180 & $0.322 / 0.334=0.965$ & 0 \\
\hline $10 / 02 / 2004$ & 192 & 0.182 & $0.060 / 0.309=0.194$ & 0 \\
\hline $24 / 03 / 2004$ & 173 & 0.069 & $0.052 / 0.265=0.197$ & 0 \\
\hline $12 / 08 / 2004$ & 52.1 & 0.085 & $0.067 / 0.348=0.193$ & 4 \\
\hline $29 / 01 / 2005$ & 865.6 & 0.101 & $0.144 / 0.480=0.300$ & 2 \\
\hline
\end{tabular}




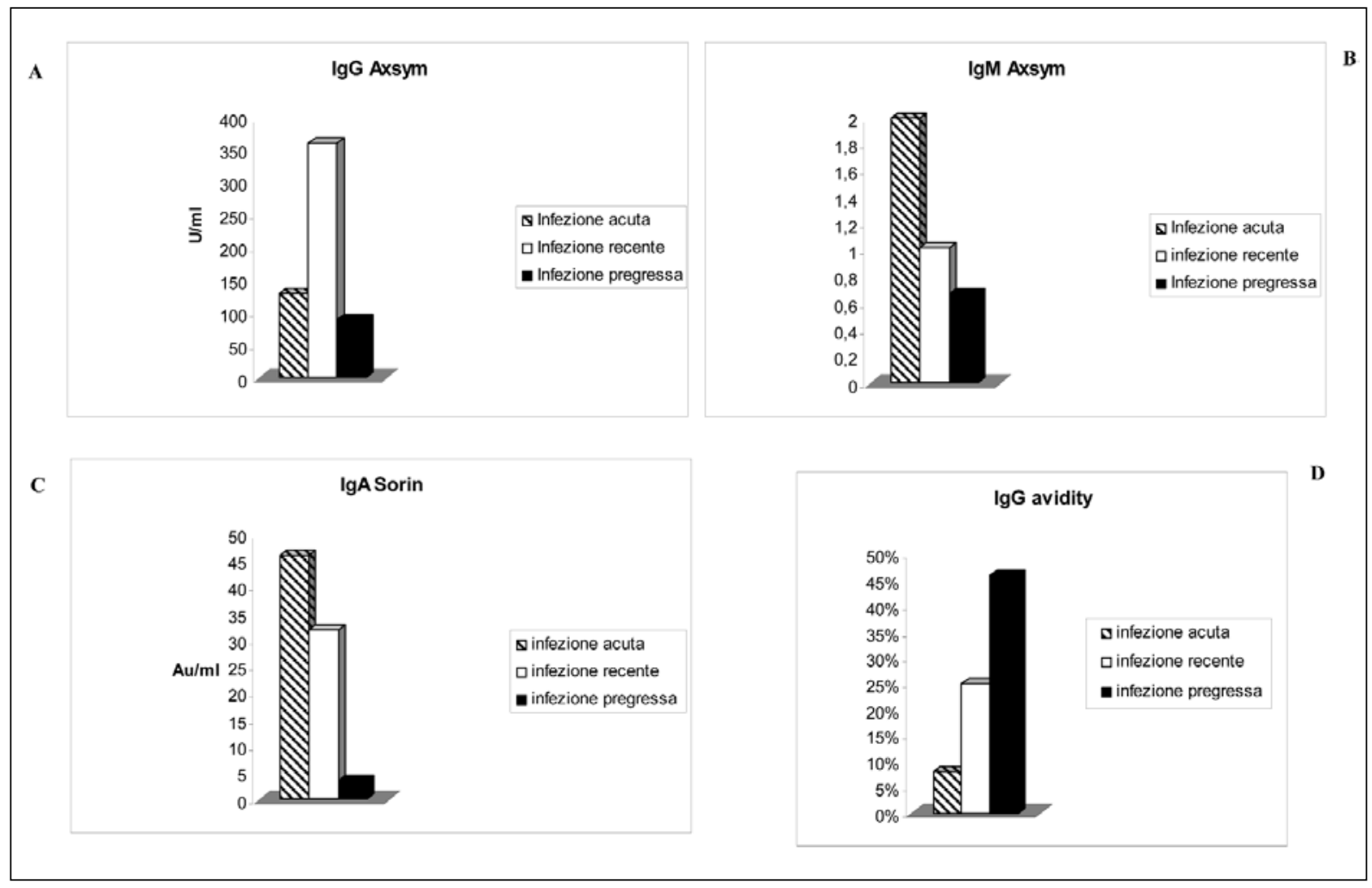

Figura I. Pattterns immunitari nelle diverse fasi dell'infezione toxoplasmica.

\section{BIBLIOGRAFIA}

1. Couvreur J. Toxoplasmose Congenitale, Epidemiologie, physiopatologie, evolution, diagnostic, prognostic, principes du traitement, prevention.Rev Prat 1992; 42: 2.

2. Dubey JP. Toxoplasmosis (Zoonosis Update) J Am Vet Med Assoc 1994; 205 (11): 1593-8.

3. Dunn D. Mother to child transmission of toxoplasmosis: risks estimates for clinical couselling. Lancet 1999; 353: 1829-33.

4. Gussetti N, D'Elia R, Mottola A, Rigoli E. Natural IgM antibodies against Toxoplasma gondii durino pregnancy. Am J Obstet And Gynecol 1990; 162: 1359-60.

5. Gutierrez-Zufiaurre N, et al. Seroprevalence of antibodies against Treponema, Toxoplasma gondii, rubella virus, hepatitis $\mathrm{B}$ and $\mathrm{C}$ virus, and HIV in pregnancy. Enferm Infecc Microbiol Clin 2004; 22: 512-6.

6. Hedman K, Lappalainen M, Soderlund M, Hedman L. Avidity $\mathrm{IgG}$ in serodiagnosis of infectious diseases. Med Microbiol 1993; 4: 123-9.

7. Lecolier B. Seroconversion de la toxoplasmose. Tempo medico 1991; 422: 13.

8. Mombro M, et al. Congenital Toxoplasmosis: 10 years follow-up. Eur J Pediatr 1995; 154: 635-9.

9. Olliaro P. Congenital toxoplasmosis. Clin Evid 2004; 12: 1058-61.

10. Patel B, Youg Y, Duffy K, Tanner RP, Johynson, J, Holliman R. A detection and the investigation of clinical toxoplasmosis. J Me. Microbiol 1993; 38: 286-92.
11. Remington, JS., Miller, MJ., Brownlee, I. IgM Antibodies in Acute Toxoplasmosis: II. Prevalence and Significance in Acquired cases. J Lab Clin Med 1968; 71: 855.

12. Sensini A, Pascoli S, Marchetti D, Castronari R, Marangi M, Sbaraglia G, et IgG avidity in the serodiagnosis of acute Toxoplasma condii infection: a multicenter study. Clin Microbiol Inf 1996: 2: 25-9.

13. Sequela JP. Cinetique des anticorps IgA, IgG, IgM dans la toxoplasmose. Lyon Med 1982: 248: 21-3.

14. Stronati M, Lanzarini P, Meroni V, Rondini G. Toxoplasmosi: prevenzione, diagnosi e terapia nella donna in gravidanza, nel feto e nel neonato. Riv Ital Ped 1996: 22: 145-53.

\author{
Alessandra Marabese \\ Azienda Sanitaria di Bolzano \\ Laboratorio Microbiologia e Virologia \\ E-mail: alessandra.marabese@asbz.it
}

